\title{
BEST APPROXIMATION IN METRIC SPACES
}

\author{
ROSHDI KHALIL
}

(Communicated by Doug W. Curtis)

\begin{abstract}
A metric space $(X, d)$ is called an $M$-space if for every $x$ and $y$ in $X$ and for every $r \in[0, \lambda]$ we have $B[x, r] \cap B[y, \lambda-r]=\{z\}$ for some $z \in X$, where $\lambda=d(x, y)$. It is the object of this paper to study $M$-spaces in terms of proximinality properties of certain sets.
\end{abstract}

0. Introduction. Let $(X, d)$ be a metric space, and $G$ be a closed subset of $X$. For $x \in X$, let $\rho(x, G)=\inf \{d(x, y): y \in G\}$. If the infimum is attained for all $x \in X$, then $G$ is called proximinal in $X$.

The problem of priximinality of subsets in normed spaces has been studied by many authors. We refer mainly to the encyclopedia of Singer [10], and other references cited there, where the problem is treated in detail. Singer suggested the problem of proximinality in the so-called convex metric spaces.

In metric linear spaces, many results on the proximinality problem were obtained in $[\mathbf{1}, \mathbf{2}]$ and other papers cited in [2].

Some results on proximinality in metric (but necessarity linear) spaces appeared in [6 and 7]. In [7], Busemann considered externally convex metric spaces $(X, d)$ $[4$, p. 51], which satisfy the following conditions:

(i) For any $x, y, \in X$ there exists $z \in X, z \notin\{x, y\}$ such that $d(x, z)+d(y, z)=$ $d(x, y)$

(ii) If $x, y, z_{1}, z_{2} \in X$ such that $d(x, y)+d\left(y, z_{1}\right)=d\left(x, z_{1}\right), d(x, y)+d\left(y z_{2}\right)=$ $d\left(x, z_{2}\right)$ and $d\left(y, z_{1}\right)=d\left(y, z_{2}\right)$, then $z_{1}=z_{2}$.

(iii) Every bounded infinite set in $(X, d)$ has an accumulation point. Busemann proved that the balls in $(X, d)$ are convex if and only if they are Chebyshev.

In this paper we prove results which relate proximinality of sets to the metric structure in the space. We prove among other things that Busemann's result is true for a larger class of metric spaces. More results on convexity and Chebyshevity of sets are presented.

In $\S 1$, we define and characterize $M$-spaces. In $\S 2$, we study the convexity of balls in relation to prominality of convex sets in $M$-spaces. Some results on proximinality in strictly convex metric spaces are obtained.

1. $M$-spaces. Let $(X, d)$ be a metric space. For $x \in X, r>0$, we let $B(x, r)=$ $\{y \in X: d(x, y)<r\}, B[x, r]=\{y \in X: d(x, y) \leq r\}$ and $S(x, r)=\{y \in$ $X: d(x, y)=r\}$. Following Menger [11], we call $(X, d)$ a convex metric space if $B\left[x, r_{1}\right] \cap B\left[y, r_{2}\right] \neq \varnothing$ whenever $r_{1}+r_{2} \geq d(x, y)$. Some of the properties of these

Received by the editors April 2, 1986 and, in revised form, October 24, 1986 and March 2, 1987.

1980 Mathematics Subject Classification (1985 Revision). Primary 51K05, 51K99.

Key words and phrases. Midpoint, $M$-space, convex set. 
spaces were introduced by Blumenthal [4]. Further results can be found in [3 and 5].

DEFinition 1.1. A metric space $(X, d)$ is called an $M$-space if for every two points $x$ and $y$ in $X$ with $d(x, y)=\lambda$, and for every $r \in[0, \lambda]$, there exists a unique $z_{r} \in X$ such that

$$
B[x, r] \cap B[y, \lambda-r]=\left\{z_{r}\right\} .
$$

If $r=\lambda / 2$, the point $z_{r}$ is called the midpoint of $x$ and $y$, and we write $z_{r}=m(x, y)$.

For $x$ and $y$ in $X$, a curve joining $x$ to $y$ in $X$ is the image under a one-to-one continuous map $\gamma$ of a closed interval $[a, b]$ into $X$ such that $\gamma(a)=x$ and $\gamma(b)=y$. We write $\gamma$ to denote the curve and the function at the same time.

If $\gamma$ is a curve joining $x$ to $y$ in $X$, then the length of $\gamma$ is defined by

$$
l(\gamma)=\lim _{\Delta_{n} \rightarrow 0} \sum_{i=1}^{n} d\left(\gamma\left(a_{i-1}\right), \gamma\left(a_{i}\right)\right)
$$

where $\Delta_{n}=\sup _{1 \leq i \leq n}\left|a_{i}-a_{i-1}\right|$, and $\left\{a=a_{0}, a_{1}, \ldots, a_{n}=b\right\}$ is a partition of $[a, b]=\left[\gamma^{-1}(x), \gamma^{-1}(y)\right]$.

If $d(x, y)=\lambda$ and if $\{z\}=B[x,(1-t) \lambda] \cap B[y, t \lambda], t \in[0,1]$, then we write $z=t x+(1-t) y$.

THE SET $G[x, y]$. Let $(X, d)$ be an $M$-space and $x, y \in X$. For each $n$, let us define a set $E(n) \subseteq X$ as follows:

$$
\begin{gathered}
E(0)=\{a(0,0)=x, a(0,1)=y\}, \\
E(1)=\{a(1,0)=x, a(1,1), a(1,2)=y\}, \quad \text { where } a(1,1)=m(x, y) .
\end{gathered}
$$

Assume that $E(n)$ has been defined such that $E(n)=\{a(n, 0)=x, a(n, 1), a(n, 2)$, $\left.\ldots, a\left(n, 2^{n}\right)=y\right\}$, where $a(n, k)=m(a(n, k-1), a(n, k+1)), 0<k<2^{n}$. Then we define $E(n+1)$ as follows

$$
E(n+1)=\left\{a(n+1,0)=x, a(n+1,1), \ldots, a\left(n+1,2^{n+1}\right)=y\right\},
$$

where

$$
a(n+1, k)= \begin{cases}m(a(n,(k-1) / 2), a(n,(k+1) / 2)) & \text { if } k \text { is odd } \\ a(n, k / 2) & \text { if } k \text { is even }\end{cases}
$$

for $0<k<2^{n+1}$. For each $n$, we have

$$
d(x, y)=\sum_{0}^{2^{n}-1} d(a(n, k), a(n, k+1))
$$

Further, for all positive integers $0<t<2^{n}$, we have

$$
d(a(n, s), a(n, t))=\sum_{k=s}^{t-1} d(a(n, k), a(n, k+1))
$$

Set $A(x, y)=\bigcup_{n=0}^{\infty} E(n)$ and $G[x, y]=\overline{A(x, y)} . G[x, y]$ is called a line segment joining $x$ to $y$. 
THEOREM 1.1. Let $(X, d)$ be an $M$-space and $x, y \in X$. Then $G[x, y]$ is a curve of minimum length joining $x$ to $y$.

ProOF. Let $d(x, y)=1$, and $Q$ be the dyadic numbers in $[0,1]$. Define the map: $\gamma: Q \rightarrow A[x, y]$ such that $\gamma(r)=a(n, k) \in E(n)$, when $r=k / 2^{n}$ and $0<k<2^{n}$.

It follows from the definition of $\gamma$ and the construction of $A(x, y)$ that $\gamma$ is an isometry from $Q$ onto $A(x, y)$. Let $t \in[0,1]$, and $t_{n}$ be a decreasing sequence in $Q$ such that $t_{n} \rightarrow t$. Since $t_{n}$ is a Cauchy sequence in $Q$ and $\gamma$ is an isometry on $Q$, it follows that $\gamma\left(t_{n}\right)$ is a Cauchy sequence in $G[x, y]$. Further, $d\left(\gamma\left(t_{n}\right), x\right)$ is a decreasing sequence. Thus $d\left(\gamma\left(t_{n}\right), x\right) \rightarrow t$. Since $(X, d)$ is an $M$-space, there exists $z \in X$ such that $B[x, t] \cap B[y, 1-t]=\{z\}$.

We claim that $\gamma\left(t_{n}\right)$ converges to $z$. We have $d\left(\gamma\left(t_{n}\right), x\right)>t$, and using the fact that $(X, d)$ is an $M$-space, we find a unique $z_{n}$ such that $B[x, t] \cap B\left[\gamma\left(t_{n}\right), r_{n}-t\right]=$ $\left\{z_{n}\right\}$, where $r_{n}=d\left(\gamma\left(t_{n}\right), x\right)$. Furthermore, $d\left(y, \gamma\left(t_{n}\right)\right)+d\left(\gamma\left(t_{n}\right), x\right)=d(x, y)$, and $d\left(\gamma\left(t_{n}\right) z_{n}\right)+d\left(z_{n}, x\right)=d\left(\gamma\left(t_{n}\right), x\right)$. It follows that $d\left(y, z_{n}\right)+d\left(z_{n}, x\right)=d(x, y)$. But $d\left(z_{n}, x\right)=t$, so $z_{n}=z$ because $(X, d)$ is an $M$-space. Since $d\left(\gamma\left(t_{n}\right), z_{n}\right) \rightarrow 0$, $\gamma\left(t_{n}\right) \rightarrow z$.

We now define $\gamma(t)=z$. This establishes the extension of $\gamma$ from $Q$ to $[0,1]$. But $\gamma$ is an isometry on $Q$. Hence $\gamma$ is continuous (and an isometry by construction) on $[0,1]$. This completes the proof of the theorem.

Now, we give a characterization of $M$-spaces.

THEOREM 1.2. Let $(X, d)$ be a metric space. Then $(X, d)$ is an $M$-space if and only if any two points $x$ and $y$ in $X$ can be joined by a unique curve of length $d(x, y)$.

ProOF. Let $(X, d)$ be an $M$-space, and $x, y \in X$, with $d(x, y)=\lambda$. By Theorem 1.1 , there is a curve of length $\lambda$ joining $x$ to $y$, namely $G[x, y]$. If possible, let $\gamma$ be another curve of length $\lambda$ joining $x$ to $y$. Since $\gamma$ is connected and $(X, d)$ is an $M$-space, it follows that $z_{t}=(1-t) x+t y \in \gamma$ for all $t \in[0,1]$. Hence $G[x, y] \subset \gamma$. But $l(\gamma)=l(G[x, y])=\lambda$. Hence $\gamma=G(x, y)$.

Conversely, let $(X, d)$ be such that any two points of $X$ are joined by a unique curve of minimum length. If $x, y \in X, d(x, y)=\lambda$, let $\gamma$ be the unique curve of length $\lambda$ joining $x$ to $y$.

Let $E(t)=B[x,(1-t) \lambda] \cap B[y, t \lambda], 0 \leq t \leq 1$. Since $\gamma$ is connected, $E_{1}(t)=$ $\gamma \cap S(x,(1-t \lambda)) \neq \varnothing, E_{2}(t)=\gamma \cap S(y, t \lambda) \neq \varnothing$. We claim that $E(t) \neq \varnothing$. For if $E(t)=\varnothing$ then there exists $z_{1} \in E_{1}$ and $z_{2} \in E_{2}$ such that $d\left(z_{1}, z_{2}\right) \geq \varepsilon>0$. The set $\left\{\gamma^{-1}(x), \gamma^{-1}\left(z_{1}\right), \gamma^{-1}\left(z_{2}\right), \gamma^{-1}(y)\right\}$ is a partition of the domain of $\gamma$. Hence

$$
l(\gamma) \geq d\left(x, z_{1}\right)+d\left(z_{1}, z_{2}\right)+d\left(z_{2}, y\right)>d(x, y)+\varepsilon .
$$

Hence $E(t) \neq \varnothing$.

Now, we claim $E(t) \subseteq \gamma$. Let $z \in E(t)$. By the hypothesis, there exist unique curves $\gamma_{1}^{t}$ and $\gamma_{2}^{t}$ joining $x$ to $z$ and $z$ to $y$, respectively, such that $l\left(\gamma_{1}^{t}\right)=d(x, z)$ and $l\left(\gamma_{2}^{t}\right)=d(z, y)$. We may assume by using a standard scaling down method that the domain of $\gamma_{1}^{t}$ is $[0,(1-t) \lambda]$, and the domain of $\gamma_{2}^{t}$ is $[(1-t) \lambda, \lambda]$. Consider the function $\gamma_{0}^{t}:[0, \lambda] \rightarrow X$, defined by

$$
\gamma_{0}^{t}(s)= \begin{cases}\gamma_{1}^{t}(s) & \text { on }[0,(1-t) \lambda] \\ \gamma_{2}^{t}(s) & \text { on }[(1-t), \lambda]\end{cases}
$$


Then $\gamma_{0}^{t}(0)=x, \gamma_{0}^{t}((1-t) \lambda)=z, \gamma_{0}^{t}(\lambda)=y$. Also $\gamma_{0}^{t}$ is continuous, and $l\left(\gamma_{0}^{t}\right)=$ $l\left(\gamma_{1}^{t}\right)+l\left(\gamma_{2}^{t}\right)=d(x, y)=l(\gamma)$. Consequently, $\gamma_{0}^{t}=\gamma$, for all $t$. Hence $E(t) \subseteq \gamma$.

Finally, $E(t)$ consists of one point for each $t \in[0,1]$. For otherwise, one can easily see that $l(\gamma)>d(x, y)$. Thus $(X, d)$ is an $M$-space. This completes the proof of the theorem.

REMARK. Blumenthal [4], proved a similar result. However, he assumed completeness and convexity of $(X, d)$. Our method of proof is different from his.

2. Best approximation in $(X, d)$. Let $(X, d)$ be a given metric space, and $G$ a closed subset of $X$. Set $b(x, G)=\{y \in G: d(x, y)=\rho(x, G)\}$. The set $G$ is called proximinal if $b(x, G) \neq \varnothing$. It is called Chebyshev if $b(x, G)$ contains only one point.

Much work has been done to determine the proximinality of closed subspaces in normed spaces. It is not our objective to work on this problem. But here we study some properties of the metric $d$ in terms of proximinality and Chebyshevity of some sets in $X$.

For $x \in G$, set $P(G, x)=\{y \in X: \rho(y, G)=d(x, y)\}$.

THEOREM 2.1. Let $(X, d)$ be a convex metric space. Then the following are equivalent

(i) $(X, d)$ is an $M$-space;

(ii) $B[z, r]$ is Chebyshev for all $z \in X, r>0$;

(iii) $P(A, x) \cap P(A, y)=\varnothing$ for $x \neq y$ and all closed balls $A$ in $X$.

Proof. (i) $\rightarrow$ (ii). Let $B[z, r]$ be any closed ball. Let $x \in X \backslash B[z, r]$ and $d(x, z)=$ $s=r+\lambda$. Then $\rho(x, B[z, r])=\lambda$. By (i), $B[x, \lambda] \cap B[z, r]=\{y\}$ for some $y \in X$. Clearly, $b(x, B[z, r])=\{y\}$ and $B[z, r]$ is Chebyshev.

(ii) $\rightarrow$ (iii). If $P(A, x) \cap P(A, y) \neq \varnothing$ for some ball $A=B[z, r]$, and some $x, y \in A$, then for $w \in P(A, x) \cap P(A, y)$

$$
d(w, x)=d(w, y)=\rho(w, A)
$$

which contradicts (ii).

(iii) $\rightarrow$ (i). Let $x, y \in X$ and $d(x, y)=\lambda$. By the convexity of $(X, d)$, there exists a $t$ such that $B[x,(1-t) \lambda] \cap B[y, t \lambda]=E(t) \neq \varnothing$. If $z_{1}, z_{2} \in E(t), z_{1} \neq z_{2}$, then

$$
x \in P\left(B[y, t \lambda], z_{1}\right) \cap P\left(B[y, t \lambda], z_{2}\right) \neq \varnothing,
$$

which contradicts (iii). Hence $(X, d)$ is an $M$-space.

A set $G \subseteq X$ is called convex if for every $x, y \in G, B[x,(1-t) \lambda] \cap B[y, t \lambda] \subseteq G$ for all $t \in[0,1]$, where $\lambda=d(x, y)$. Set

$$
L(x, y)=\bigcup_{0 \leq t \leq 1}(B[x,(1-t) \lambda] \cap B[y, t \lambda])=\bigcup_{0 \leq r \leq \lambda}(B[x, r] \cap B[y, \lambda-r]) .
$$

REMARK. It follows from the construction of $G[x, y]$ and the proof of Theorem 1.1 that $G[x, y]=L(x, y)$.

THEOREM 2.2. Let $(X, d)$ be an $M$-space. The following are equivalent

(i) Closed balls in $(X, d)$ are convex;

(ii) If $G$ is a closed convex set in $(X, d)$ and $x \notin G$, then $b(x, G)$ is convex. 
Proof. (i) $\rightarrow$ (ii). Let $G$ be a closed convex set in $(X, d)$ and $x \notin G$. If $b(x, G)=$ $\varnothing$ then it is trivially convex. So, let $z_{1}, z_{2} \in b(x, G)$ and $d\left(z_{1}, z_{2}\right)=\lambda$. If $\rho(x, G)=$ $r$, then $z_{1}, z_{2} \in B[x, r]$. Since closed balls are assumed to be convex then $G\left[z_{1}, z_{2}\right] \subseteq$ $B[x, r]$. Since $G$ is convex, we get $G\left[z_{1}, z_{2}\right] \subseteq G$. Consequently, $G\left[z_{1}, z_{2}\right] \subseteq S(x, r)$. Hence $G\left[z_{1}, z_{2}\right] \subseteq b(x, G)$.

(ii) $\rightarrow$ (i). Let $B[z, r]$ be any closed ball in $(X, d)$ and $x, y \in B[z, r]$. If possible assume that for some $t \in[0,1],\{B[x,(1-t) \lambda] \cap B[y, t \lambda]\} \not \subset B[z, r]$, where $\lambda=$ $d(x, y)$. By Theorem 1.2, the points $x$ and $y$ can be joined by a unique curve of length $\lambda=d(x, y)$. If $\gamma$ is this curve, then again by Theorem $1.2, \gamma$ is a convex closed set in $(X, d)$.

By the connectedness of $\gamma$, there exists at least two points $z_{1}$ and $z_{2}$ such that $\left\{z_{1}, z_{2}\right\} \subseteq \gamma \cap S(z, r)$. Then $z_{1}, z_{2} \in b(z, \gamma)$. However, $G\left[z_{1}, z_{2}\right] \nsubseteq b(z, \gamma)$. This contradicts (ii). Thus $B[z, r]$ must be convex.

THEOREM 2.3. Let $(X, d)$ be an $M$-space in which every proximinal convex set is Chebyshev. Then $B[z, r]$ is convex for all $z \in X$ and $r>0$.

PROOF. Let $x, y \in B[z, r], d(x, y)=\lambda$. If possible, let $\{B[x,(1-t) \lambda] \cap B[y, t \lambda]\} \notin$ $B[x, r]$ for some $t \in[0,1]$. Then as in the proof of Theorem 2.2 , there exist distinct points $z_{1}, z_{2} \in S[z, r]$ such that $\gamma=G\left[z_{1}, z_{2}\right]$ is not contained in $B[z, r]$. Since $\gamma$ is compact (being the continuous image of $\left[0, d\left(z_{1}, z_{2}\right)\right]$ ) it is easily seen that $\gamma$ is proximinal. But $z_{1}, z_{2} \in b(z, \gamma)$, contradicting the Chebyshevity of $\gamma$, since $\gamma$ is convex. Hence $B[z, r]$ is convex.

A convex metric space $(X, d)$ is called strictly convex if, for $x, y \in B[z, r]$ with $d(x, y)=\lambda$, then $B[x,(1-t) \lambda] \cap B[y, t \lambda] \subseteq B(z, r)$ for all $t \in(0,1)$, and all $z \in X, r>0$. For normed linear spaces, it was proved in [9] that strict convexity characterizes $M$-spaces. This was generalized to uniform convexity in [8]. Here we can prove:

THEOREM 2.4. Let $(X, d)$ be a strictly convex metric space. Then $(X, d)$ is an $M$-space.

Proof. Let $x, y \in X$ and $d(x, y)=\lambda$. By the convexity of $(X, d), E(t)=$ $B[x,(1-t) \lambda] \cap B[y, t \lambda] \neq \varnothing$ for all $t \in(0,1)$. If possible let $z_{1}, z_{2} \in E(t)$. By strict convexity of $(X, d)$ we get

$$
\left.B\left[z_{1},(1-s) \xi\right] \cap B\left[z_{2}, s \xi\right] \subseteq B[x,(1-t) \lambda)\right] \cap B[y, t \lambda]
$$

for all $s \in(0,1)$, where $\xi=d\left(z_{1}, z_{2}\right)$. This can happen only if $z_{1}=z_{2}$. Hence $(X, d)$ is an $M$-space.

Chebyshevity of proximinal convex sets in strictly convex metric linear space was proved in $[\mathbf{1}]$. We prove the same result in our setting.

THEOREM 2.5. Let $(X, d)$ be a strictly convex metric space. Then every proximinal convex set in $(X, d)$ is Chebyshev.

ProOF. Let $G \subseteq X$ be proximinal and convex. If possible let $z \in X \backslash G$ such that $b(z, G)$ contains more than one element. Let $\rho(z, G)=r$, and consider $\left\{z_{1}, z_{2}\right\} \subseteq$ $b(z, G)$. Let $d\left(z_{1}, z_{2}\right)=\lambda$. Since $\left\{z_{1}, z_{2}\right\} \subseteq B[z, r]$, it follows from the strict convexity of $(X, d)$ that

$$
w(t)=B\left[z_{1},(1-t) \lambda\right] \cap B\left[z_{2}, t \lambda\right] \in B(z, r) .
$$


The convexity of $G$ implies that $w(t) \in G$. This is impossible [10]. Thus $z_{1}=z_{2}$, and $G$ is Chebyshev.

Set $G(x, y)=G[x, y] \backslash[x, y]$. A point $w \in S(z, r)$ is called an extreme point of $B[z, r]$ if $w \in G(x, y)$ for all $x, y \in B[z, r]$. The following characterization of strict convexity is immediate (but useful).

THEOREM 2.6. Let $(X, d)$ be a convex metric space. The following are equivalent:

(i) $(X, d)$ is strictly convex;

(ii) Every point of $S(z, r)$ is an extreme point of $B[z, r]$ for all $z \in X$ and $r>0$;

(iii) $S(z, r)$ does not contain any line segment.

Using these theorems one obtains a stronger version of Theorem 2.3.

THEOREM 2.7. Let $(X, d)$ be an $M$-space. Then the following are equivalent:

(i) $(X, d)$ is strictly convex;

(ii) Every proximal convex set is Chebyshev.

PrOOF. It suffices to show that if every proximinal convex set is Chebyshev, then every closed ball is strictly convex. Let $B[z, r]$ denote any such ball and suppose it is not strictly convex. Then by Theorem 2.6, there exist three points $u, v, w$ that are elements of $S(z, r)$ such that $v \in G[u, w]$. Then as in the proof of Theorem $2.3, G[u, w]$ is proximinal. Then $G[u, w]$ is Chebyshev and thus by connectedness of $G[u, w]$ and continuity of the metric, there exist points $x, y \in G[u, w]$ such that $v \in G[x, y]$ with $d(z, x)=d(z, y)=k<d(z, v)$. Thus $v \notin B[z, k]$ and hence $B[z, k]$ is not convex, contrary to Theorem 2.3 .

3. Results in strong $M$-spaces. In light of the fact that every $M$-space is convex and every strictly convex metric space is an $M$-space, one might conjecture that every $M$-space is strictly convex. That this is not the case is shown by the following example.

Let $(X, c)$ be a closed ball of the $S_{2, r}[4$, pp. 16-18] of radius $\rho$ with $\pi r / 4<\rho<$ $\pi r / 2$. Since $X$ is convex and contains no diametral point pairs of the $S_{2, r}, X$ is an $M$-space. However, letting $x, y$ denote points of $X$ such that $d(x, y)=d(x, z)=$ $\pi r / 2$, then all points $w$ of $X$ between $y$ and $z$ have the property that $d(x, w)=\pi r / 2$ contradicting strict convexity.

DEFINITION 3.1. An $M$-space $(X, d)$ is called a strong $M$-space if for every two points $x, y$ in $X$ with $d(x, y)=\lambda$ and for every positive real number $r \neq \lambda$, there exists a unique $z_{r}$ such that $S[x, r] \cap S[y,|\lambda-r|]=\left\{z_{r}\right\}$.

DEFINITION 3.2. A metric space is called externally convex if for all distinct points $x, y$ such that $d(x, y)=\lambda$, and $k>\lambda$ there exists a unique $z$ of $X$ such that $d(x, y)+d(y, z)=d(x, z)=k$.

It is clear that not every convex metric space is externally convex. It may be noted that not every externally convex metric space is convex, as is illustrated by the following example.

Let the point set of $(X, d)$ be the union of the two lines in the cartesian plane whose equations are $y=1$ and $y=2$. Let the distance $d\left(p_{1}, p_{2}\right)$ for $p_{1}=\left(x_{1}, y_{1}\right)$ and $p_{2}=\left(x_{2}, y_{2}\right)$ be given by $\left|x_{1}-x_{2}\right|$ if $y_{1}=y_{2}$ and by $1+\left|x_{1}\right|+\left|x_{2}\right|$ if $y_{1} \neq y_{2}$. This satisfies the condition of external convexity but convexity fails when applied to the points $(0,1)$ and $(0,2)$. 
It may be further noted that not every externally convex $M$-space is a strong $M$-space. Such a counterexample is constructed from three distinct half-lines of the euclidean plane with common origin $O$. The metric of points $x, y$ in the space is defined to be their euclidean distance if they lie on the same half-line, but their distance is defined to be the sum of their respective distances from $O$ if they lie on different half-lines.

Using the method of proof of Theorem 1.1 and Theorem 1.2, one obtains

THEOREM 3.1. For any two points $x, y$ of a strong $M$-space and real numbers $k^{\prime}, k^{\prime \prime}>d(x, y)$ there exist unique points $u, v$ such that $G[x, y]$ is contained in $G[u, v]$ with $d(x, u)=k^{\prime}$ and $d(y, v)=k^{\prime \prime}$.

THEOREM 3.2. For any two points $x, y$ of a strong $M$-space $(X, d)$ there exists exactly one set $L(x, y)$ containing $x, y$ such that $L(x, y)$ is congruent to the euclidean line.

PROOF. The theorem follows by using Theorem 3.1 a denumerable number of times, extending the partial congruence each time, as the $k^{\prime}, k^{\prime \prime}$ become large without limit.

LEMMA 3.1. Let $(X, d)$ be any metric space and $G$ be a proximal subset of $(X, d)$. Let $x \in X \backslash G$. If $z \in b(x, G)$, then $z \in b(y, G)$ for all $y \in G(x, z)$.

ProOF. If possible let $z \in b(y, G)$ for some $y \in G(x, z)$. Thus $d(y, w)<d(y, z)$ for $w \in b(y, G)$.

This implies that

$$
d(x, w) \leq d(x, y)+d(w, y)<d(x, y)+d(y, z)=d(x, z) .
$$

This is a contradiction.

REMARK. Lemma 3.1 is similar to Lemma 2.1 in [10] for normed linear spaces.

Let $x, y$ be any two elements in an $M$-space $(X, d)$. We let $G(x, y,-)$ denote the largest line segment containing $G(x, y)$ for which $x$ is an extreme point.

A set $G \subseteq(X, d)$ is called a sun if for all $x \in X \backslash G$ and $y \in b(x, G)$, one has $y \in b(z, G)$ for all $z \in G(y, x,-)$. This is similar to the concept of sun in normed linear spaces.

THEOREM 3.3. Every closed ball in a strong $M$-space $(X, d)$ is a sun.

ProOF. Let $B[z, r]$ be any closed ball in $(X, d)$ and $x \in X \backslash B[z, r]$. From Theorem 2.1, $B[z, r]$ is Chebyshev. Hence $b(x, B[z, r])=\{y\}$ for some $y \in S(z, r)$. If $w \in G(y, x)$, then by Lemma 3.1, $\{y\}=b(w, B[z, r])$. So let $w \in G(y, x,-)$ be such that $d(w, y)>d(w, x)$. But from the definition of $G(y, x,-), d(w, y)=$ $d(w, x)+d(x, y)$, and by Theorem 3.2, $d(z, w)=d(z, y)+d(y, w)$. Hence $\{y\}=$ $b(w, B[z, r])$ and $B[z, r]$ is a sun.

In an $M$-space, it is not the case that every convex set is a sun. The closed ball of $S_{2, r}$ considered earlier in this paper in which $G$ is a connected portion of a great circle contained, together with its pole, in the interior of the defining closed ball, is such a set. It is an open question whether every closed ball in an $M$-space is a sun.

Part of this research was done while the author was on sabatical at Univ. of Michigan. 
ACKNOWLEDGEMENTS. The author would like to thank the referee for very helpful comments and suggestions. $\S 3$ of this paper was shaped up by the referee. In particular, the introduction of Definitions 3.1, 3.2, Theorems 3.1, 3.2 and the present form of Theorem 3.3 are due to the referee.

\section{REFERENCES}

1. G. Ahuja et. al., Best approximation on convex sets in metric linear spaces, Math. Nachr. 78 (1977), 125-130.

2. G. Albinus, Approximation in metric linear spaces, Approximation Theory, Banach Center Publ., vol. 4, 1979, pp. 7-18.

3. N. Aronszajn and P. Panitchapakdi, Extension of uniformly continuous transformations and hyper convex metric spaces, Pacific J. Math. 6 (1956), 405-439.

4. L. M. Blumenthal, Distance geometry, Clarendon Press, Oxford, 1953.

5. A. Berard, Characterizations of metric spaces by the use of their midsets intervals, Fund. Math. 73 (1971), 1-7.

6. H. Busemann, Metric methods in Finsler spaces and in the foundations of geometry, Ann. of Math. Studies, no. 8, Princeton Univ. Press, Princeton, N.J., 1942.

7. __ Note on a theorem on convex sets, Mat. Tidsskr. B (1947), 32-34.

8. S. Izumino, Khalil's theorem and a property of uniformly convex spaces, Math. Rep. Toyama Univ. 6 (1983), 41-46.

9. R. Khalil, Extreme points of the unit ball of Banach spaces, Math. Rep. Toyama Univ. 4 (1981), 41-45.

10. I. Singer, Best approximation in normed linear spaces by elements of linear subspaces, Springer, New York, 1970.

11. K. Menger, Untersuchungen über allegemeine Metrik, Math. Ann. 100 (1928), 75-163.

Department of Mathematics, KuWait University, P. O. BoX 5969, KuWAit 